\title{
A new technique for ice-fabric analysis
}

\author{
L. A. WiLEN \\ Department of Physics and Astronomy, Ohio University, Athens, Ohio 45701, U.S.A.
}

\begin{abstract}
A new method for ice-fabric analysis which is easily automated is described. The technique relies on a set of digital images of an ice thin section viewed through crossed polarizers and rotated to various angles. From the acquired set of images, the $c$-axis orientation of all the grains in the image can be determined. The theoretical basis for the technique is described in detail, including corrections for refraction, as well as other corrections for off-axis grains and polarization/reflection effects. An experiment was performed comparing results of the new technique with those of the standard technique. Excellent agreement was obtained. It is expected that an automated system using this technique (currently almost complete) will have a large impact on the amount of information and physical knowledge which can be extracted from ice fabrics in the future.
\end{abstract}

\section{INTRODUCTION}

Glaciologists conduct a wide range of studies to address many local issues and two overarching global problems: the future of sea-level change and the history of climate change. Many of these studies require characterization of polar ice, and especially of the $c$-axis fabric of the ice.

Ice flow changes the size, shape and arrangement of crystals (also called grains) in ice, so that these crystals contain a history of the ice flow over a time interval that depends on deformation rate and temperature (e.g. Azuma and Higashi, 1985; Alley, 1992; Anandakrishnan and others, 1994). The size, shape and arrangement of grains also affect the rate and pattern of ice deformation, providing a feedback on ice flow (e.g. Budd and Jacka, 1989).

An understanding of the detailed relationship between ice microstructure and flow is of major importance because ice flow can disrupt the continuity of paleoclimatic records. For example, in ice younger than approximately 100000 years, the paleoclimatic records from the parallel Greenland Ice Sheet Project II (GISP2) and Greenland Ice Core Project (GRIP) deep ice cores through central Greenland are almost indistinguishable. In older ice, however, it is not even clear how the two records are related (Grootes and others, 1993; Taylor and others, 1993). A joint examination of both cores by GRIP and GISP2 investigators showed that ice-flow processes have disturbed the climatic records in both (Alley and others, 1995).

Ice-flow models that include crystallographic anisotropy are needed in order to fully characterize ice flow, allow calculation of depth-age scales for ice cores and recognition of flow changes affecting climatic records and allow prediction of ice-sheet response to climatic perturbation and its contribution to sea-level change.

In order to provide sufficient experimental input for the development and testing of such models, more and improved data on ice fabric are required. Crystallographic anisotropy may be measured in several ways. The $c$ axis is deformationally, optically, sonically and electrically unique in an ice crystal. This allows use of various techniques including seismic (e.g. Bentley, 1971; Blankenship and Bentley, 1987), sonic (e.g. Kohnen and Gow, 1979; Herron and others, 1985; Anandakrishnan and others, 1994) and radar experiments (e.g. Liu and others, 1994) to characterize average $c$-axis fabrics over some volume of a glacier. However, these techniques typically lack the spatial resolution to observe the grain-scale processes that reveal active physical processes and help us gain a process understanding of ice deformation (e.g. Alley and others, 1997). X-ray techniques (Mori and others, 1985) have not gained general acceptance or achieved ease of use. Thus, optical studies of $c$ axes in thin sections still dominate the field. Semi-automatic techniques involving computerized recording of data first gathered in traditional ways have been tried (Morgan and others, 1984; Lange, 1988), and a novel approach involving conoscopic viewing and smaller thin sections than are typical has been under development for well over a decade (personal communications to R. B. Alley, from T. H. Jacka and D. S. Russell-Head, 1987, 1994, 1997, 1999), but practical applications are still almost entirely based on the traditional Rigsby stage (Rigsby, 1951), as standardized and described by Langway (1958).

The current technology for determining ice fabric is extremely cumbersome and subject to inaccuracies. In the use of a Rigsby stage, the observer affixes a thin section of ice to a large, modified universal stage (Berek, 1923), sits in a cold room or at a field site, conducts a series of manipulations to bring the $c$ axis to either a vertical position or a horizontal position in the east-west plane of the device and determines which of these two applies. The observer then records which position was reached, and the rotation angles that were required to reach this position. This measurement is then repeated, typically for 100-200 grains. Because of the noncoaxiality of the stage, the manipulations move the grain left, right, towards and away from the observer, and it is incumbent on the observer to follow the grain so that the lineof-sight through a grain remains perpendicular to the base plane of the stage.

Data collected must then be corrected for refraction errors (Kamb, 1962), converted to orientation information, plotted and analyzed. Morgan and others (1984) reported that use of 
a computer logging system on a manual stage reduced typical time per thin section from 1 day to $2-3$ hours; other workers may be faster or slower. Adding time for rerunning selected samples to determine accuracy, and taking occasional coffee breaks during defrost cycles in a freezer, this is a frightfully time-consuming activity. Data are sparse, and re-analyses are seldom mentioned in the literature. Almost no data are available on the spatial distributions of $c$ axes, nearest-neighbour relations, etc.

An additional issue is related to accuracy of measurements. When properly applied, the technique is capable of achieving accuracy of a few degrees in $c$-axis orientation (Langway, 1958). Some studies have apparently fallen short of this ideal, however (as suggested by, e.g., Lipenkov and others, 1989).

Clearly, this is the sort of measurement that is ripe for automation. Automation offers the possibility of increased speed and increased accuracy. The history of glaciological research shows clearly that the transition from manually, laboriously collected data to digitally collected and analyzed data has greatly improved the scientific return. This is evident, for example, in the use of synthetic-aperture radar interferometry, repeat imagery and stop-and-go global positioning system surveying to augment or replace traditional surveying. The greatly increased datasets allow results that would be impossible with fewer data (e.g. MacAyeal and others, 1995).

In this paper, a new technique for ice-fabric analysis is described which will permit the process to be completely automated in a straightforward way.

\section{METHOD}

The technique relies on an imaging system which provides a high-resolution digital image of the ice, viewed with white light between crossed polarizers. Gray-scale images are recorded as the polarizers are rotated in unison (remaining crossed) with the sample fixed in various positions. Fueten (1997) has demonstrated the usefulness of keeping the sample fixed (as the polarizers rotate) for image-processing applications. Earlier work (Zagorodnov and others, 1994) explored briefly the idea that some $c$-axis information could be obtained by measuring the intensity of polarized light passing through an ice crystal as a function of incidence angle.

The $c$-axis orientations are determined by computer analysis of a particular set of images. In describing the technique, it will be useful to follow the terminology used by Langway (1958) to refer to the various axes of the universal stage:

$$
\begin{aligned}
& \mathrm{A}_{1}=\text { inner vertical axis } \\
& \mathrm{A}_{2}=\text { north-south axis } \\
& \mathrm{A}_{4}=\text { east-west axis } \\
& \mathrm{A}_{5}=\text { outer vertical axis (axis of the stage) }
\end{aligned}
$$

For reference, these are shown in Figure 1. Adopting Kamb's notation (Kamb, 1962), a rotation about $\mathrm{A}_{4}$ is specified by the angle $\gamma$, and a rotation about $\mathrm{A}_{2}$ by the angle $\xi$. We denote a rotation about $A_{1}$ by the angle $\phi_{\text {in }}$. The recorded images are divided into four sequences, produced in the following way:

(1) Initially, the ice thin section is oriented perpendicular to the light path, and a sequence of images is recorded as
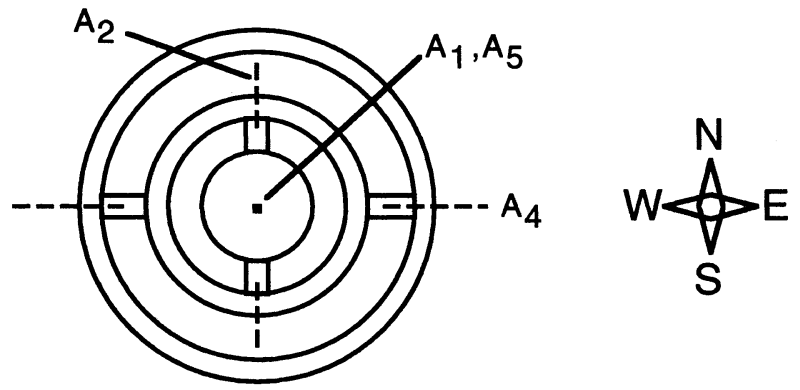

Fig. 1. Schematic diagram of the axes of the universal stage, following the notation of Langway (1958). $A_{1}$ is the axis of the innermost ring and is always perpendicular to the sample. $A_{5}$ is perpendicular to the fixed outermost ring.

the polarizers are rotated from $0^{\circ}$ through $100^{\circ}$ in $5^{\circ}$ increments.

(2) The section is tipped by $45^{\circ}$ about the north-south horizontal axis $\left(\mathrm{A}_{2}\right)$, and a second sequence of images is acquired in the same way as before.

(3) This is repeated with the section tipped by $45^{\circ}$ about the east-west $\left(\mathrm{A}_{4}\right)$ horizontal axis.

(4) Step 2 is repeated with the section rotated about the inner vertical axis $\left(A_{1}\right)$ by $-45^{\circ}$.

The settings for the four sequences are summarized below:

$$
\begin{aligned}
& \text { Sequence 1: } \gamma=0^{\circ}, \xi=0^{\circ}, \phi_{\text {in }}=0^{\circ} \\
& \text { Sequence 2: } \gamma=0^{\circ}, \xi=45^{\circ}, \phi_{\text {in }}=0^{\circ} \\
& \text { Sequence 3: } \gamma=45^{\circ}, \xi=0^{\circ}, \phi_{\text {in }}=0^{\circ} \\
& \text { Sequence 4: } \gamma=0^{\circ}, \xi=45^{\circ}, \phi_{\text {in }}=-45^{\circ}
\end{aligned}
$$

The position of the ice sample for each of the four sequences is shown schematically in Figure 2. All of the information regarding the ice fabric can be extracted from these digital images. For each sequence, there is an angle of the polarizers for each grain for which there is a minimum in transmitted light, commonly referred to as the angle of extinction. Because extinction actually occurs at four angles $\left(90^{\circ}\right.$ apart $)$ as the crossed polarizers are rotated through $360^{\circ}$, one of these angles is guaranteed to be in the $0-100^{\circ}$ range. The exact position of extinction is determined accurately and objectively by a parabolic fit to the polarizer angles with the three lowest transmitted intensities.

\section{PRINGIPLE OF TEGHNIQUE}

The basic principle of the method is as follows: The angle of extinction for one grain specifies the plane in which the $c$ axis of the grain resides (up to a $90^{\circ}$ degeneracy, which we will return to later). The second sequence, with the grain tilted, will again pick out the plane of the $c$ axis, but this

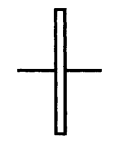

seq. 1

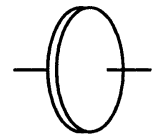

seq. 2

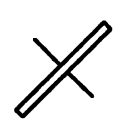

seq. 3

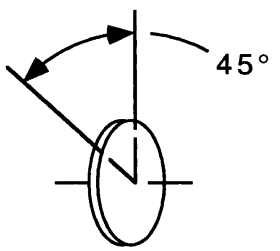

seq. 4
Fig. 2. Side view of the ice thin section corresponding to the four sequences described in the text. 
second plane will be tilted by $45^{\circ}$ with respect to the thin section. The intersection of the two planes will be a line which specifies the $c$-axis direction. The idea is very closely related to tomographic methods used in medical imaging.

In the absence of degeneracies, only two sequences would be required to specify a particular grain orientation. In fact, the true situation is more complex. The angle of extinction for any sequence is determined only up to $90^{\circ}$, yielding two planes which may contain the $c$ axis. Since the first sequence yields two planes and the second sequence yields two additional planes, the intersection of these yields four possible directions for the $c$ axis. (It is not necessary to consider the intersection of the two planes from the same sequence. Such an intersection is a line which will not, in general, lie on one of the planes from the other sequences.) The third sequence serves to distinguish between these possibilities. In the most generic case, out of the four possible $c$-axis directions, only one will lie in one of the two planes specified by the third sequence. The fourth sequence is performed as a check, but is also needed in certain special cases when the first three are insufficient to fully determine the orientation. (This can occur, for example, when the $c$ axis is aligned along the north-south direction). As will be pointed out later, it may be necessary to add a fifth sequence, or at least modify the angular settings of the four sequences, to uniquely determine all $c$-axis directions.

As with the usual technique, it is necessary to put in corrections for refraction, which have been worked out in detail for the method proposed. The theoretical details of the technique will be discussed in the following section.

\section{THEORY}

In this section, we derive the theory required to determine the predicted polarizer extinction angle for each of the sequences described above, given any specified direction for the $c$ axis of a grain. The condition for extinction will be equivalent to that due to Kamb (1962), but the technique to find the extinction angles will follow a different line of reasoning. As a check of the method, we will first re-derive the Kamb corrections for the standard technique.

\section{Refraction corrections for standard technique}

To maintain consistency, we will follow Langway's notation for the axes of the universal stage, and Kamb's notation for the rotation angles about the various axes as described above. However, since extensive use will be made of rotation matrices, it will be convenient to define a coordinate system based on $x, y$ and $z$ axes. Let us assume that light originating at the light source is traveling in the positive $z$ direction. North is identified as the direction of the $y$ axis, and east as the direction of the $x$ axis. These axes are defined in the fixed frame of the laboratory. We further define three coordinate axes in the frame of the thin section, which are denoted by $x^{\prime}, y^{\prime}$ and $z^{\prime}$. Before any rotations of the thin section are performed, the unprimed and prime axes coincide.

With this notation, we can identify a rotation about the inner vertical stage $\left(\mathrm{A}_{1}\right)$ as a rotation about the $z^{\prime}$ axis. The rotation angle $\gamma$ refers to a rotation about the $x$ axis $\left(\mathrm{A}_{4}\right)$, and the rotation angle $\xi$ is a rotation about the $y^{\prime}$ axis $\left(\mathrm{A}_{2}\right)$.

A detailed description of the standard technique using a Rigsby stage (Rigsby, 1951) can be found in Langway (1958).
A Rigsby stage consists of a universal stage (containing the ice section) sandwiched between two crossed polarizers. The first polarizer is typically oriented with its polarization direction along the north-south axis (or $y$ axis). Briefly, one first orients the $c$ axis of a given grain to lie in the east--west plane. Then the sample is rotated by the angle $\gamma$ to bring the grain out of extinction. A final rotation by the angle $\xi$ brings the grain back into extinction. After $\gamma$ is reset to $0^{\circ}$, a check is performed to determine whether the $c$ axis is oriented in a horizontal (equatorial) or vertical (polar) direction. The various angles required to bring the grain to extinction determine the unique $c$-axis direction.

Light polarized along the $y$ axis, traveling in the positive $z$ direction, will be refracted upon entering the thin section. The new direction and polarization inside the ice sample will be denoted by unit vectors $\hat{r}$ and $\hat{p}$, respectively. The direction of the $c$ axis of a grain will be denoted by the unit vector $\hat{c}$. The following condition for extinction is equivalent to that stated in Kamb's (1962) paper: $\hat{r}$ and $\hat{c}$ determine a plane. If $\hat{p}$ either lies in this plane or is perpendicular to it, then extinction will occur. Our first goal, then, will be to find expressions for $\hat{r}$ and $\hat{p}$.

\section{Calculation of $\hat{r}$}

After a rotation of the sample by the angles $\gamma$ and $\xi$, the $z^{\prime}$ axis (which is always perpendicular to the plane of the section) will be related to the $z$ axis by the polar angles $\Theta$ and $\phi$, as shown in Figure 3a. (Here, we deviate slightly from the convention of Kamb (1962). Our $\phi$ is equivalent to his $-\phi$.) Thus the $z^{\prime}$-axis direction can be written as:

$$
\hat{z}^{\prime}=(\sin \Theta \cos \phi) \hat{x}+(\sin \Theta \sin \phi) \hat{y}+(\cos \Theta) \hat{z},
$$

where $\hat{x}, \hat{y}$ and $\hat{z}$ are unit vectors along the $x, y$ and $z$ directions, respectively. One can also construct the formal rotation matrix $M$ which represents the rotations of the sample by $\gamma$ and $\xi$ corresponding to a given sequence. This is given by:

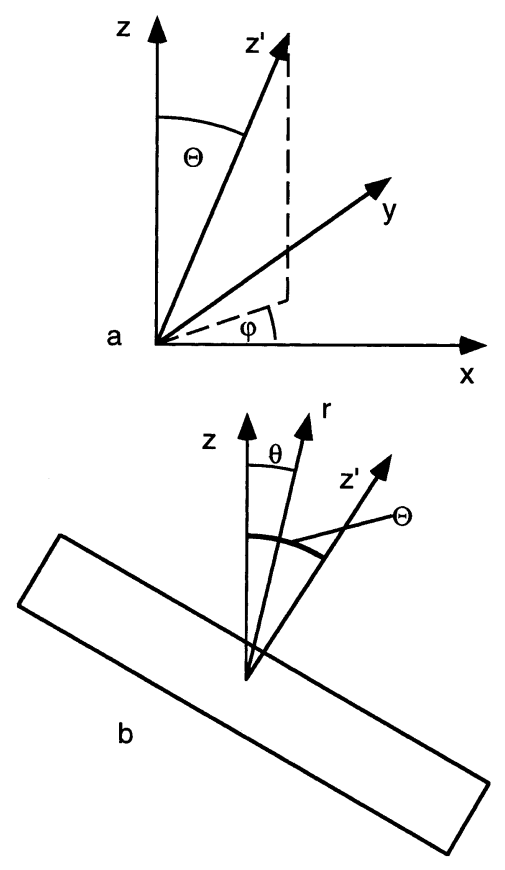

Fig. 3. (a) The polar angles $\Theta$ and $\phi$ describing the orientation of the sample. (b) Diagram of the angular relationship between the refracted-ray direction and the $z$ and $z^{\prime}$ axes, all of which lie in the plane of incidence. 


$$
M=\left(\begin{array}{ccc}
\cos \xi & 0 & \sin \xi \\
\sin \gamma \sin \xi & \cos \gamma & -\sin \gamma \cos \xi \\
-\cos \gamma \sin \xi & \sin \gamma & \cos \gamma \cos \xi
\end{array}\right)
$$

This matrix, applied to $\hat{z}$, determines $\hat{z}^{\prime}$ in terms of the laboratory coordinates (i.e. $M$ is the matrix which rotates $\hat{z}$ into $\hat{z}^{\prime}$. The result is:

$$
\hat{z}^{\prime}=(\sin \xi) \hat{x}-(\sin \gamma \cos \xi) \hat{y}+(\cos \gamma \cos \xi) \hat{z} .
$$

Comparing Equations (1) and (3), the following expressions are obtained relating the angles $\Theta$ and $\phi$ to $\gamma$ and $\xi$ :

$$
\begin{aligned}
\tan \phi & =-\sin \gamma \cot \xi \\
\cos \Theta & =\cos \gamma \cos \xi .
\end{aligned}
$$

The refracted ray, denoted by $\hat{r}$, will lie in the plane determined by the $z-z^{\prime}$ axes, commonly called the plane of incidence. We can find the refracted ray in the primed coordinate system by first constructing the rotation matrix $M^{-1}$, the inverse of the matrix defined in Equation (2):

$$
M^{-1}=\left(\begin{array}{ccc}
\cos \xi & \sin \gamma \sin \xi & -\cos \gamma \sin \xi \\
0 & \cos \gamma & \sin \gamma \\
\sin \xi & -\sin \gamma \cos \xi & \cos \gamma \cos \xi
\end{array}\right) .
$$

Applying this matrix to $\hat{z}^{\prime}$ determines $\hat{z}$ in terms of the primed coordinates:

$$
\hat{z}=-(\cos \gamma \sin \xi) \hat{x}^{\prime}+(\sin \gamma) \hat{y}^{\prime}+(\cos \xi \cos \gamma) \hat{z}^{\prime} .
$$

Note that the angle $\Theta$ between $\hat{z}$ and $\hat{z}^{\prime}$, and the angle $\theta$ between $\hat{r}$ and $\hat{z}$, are related by Snell's law (see Fig. $3 \mathrm{~b}$ ):

$$
\sin \theta=\frac{\sin \Theta}{m},
$$

where $m$ is the index of refraction of ice. Because $\hat{r}, \hat{z}$ and $\hat{z}^{\prime}$ all lie in the same plane, $\hat{r}$ and $\hat{z}$ must project down to the same azimuthal angle in the $x^{\prime}-y^{\prime}$ plane. Also, because the projection of $\hat{r}$ into this plane is proportional to $\sin \theta$, and the projection of $\hat{z}$ is proportional to $\sin \Theta$, the $x^{\prime}$ and $y^{\prime}$ coordinates of $\hat{r}$ are equal to the $x^{\prime}$ and $y^{\prime}$ coordinates of $\hat{z}$, each divided by $m$ :

$\hat{r}=\frac{-\cos \gamma \sin \xi}{m} \hat{x}^{\prime}+\frac{\sin \gamma}{m} \hat{y}^{\prime}+\frac{\sqrt{m^{2}-1+\cos ^{2} \xi \cos ^{2} \gamma}}{m} \hat{z}^{\prime}$.

To find the $z^{\prime}$ component we have used the fact that $\hat{r}$ is a unit vector.

\section{Calculation of $\hat{p}$}

To find the polarization direction of the refracted ray, we note the following: Any component of the polarization which is perpendicular to the plane of incidence will remain so upon refraction. Motivated by this fact, we construct an orthonormal basis in the primed coordinates which consists of the refracted-ray direction, a polarization direction perpendicular to the plane of incidence, denoted by $\hat{p}_{\perp}$, and a polarization direction in the plane of incidence, denoted by $\hat{p}_{\|} \cdot \hat{p}_{\perp}$ must have a vanishing $\hat{z}^{\prime}$ component and is therefore given by:

$$
\hat{p}_{\perp}=\frac{\sin \gamma}{\sqrt{1-\cos ^{2} \xi \cos ^{2} \gamma}} \hat{x}^{\prime}+\frac{\sin \xi \cos \gamma}{\sqrt{1-\cos ^{2} \xi \cos ^{2} \gamma}} \hat{y}^{\prime} .
$$

$\hat{p}_{\|}$is then found to be:

$$
\begin{aligned}
\hat{p}_{\|} & =\frac{\cos \gamma \sin \xi}{m} \sqrt{\frac{m^{2}-1+\cos ^{2} \xi \cos ^{2} \gamma}{1-\cos ^{2} \xi \cos ^{2} \gamma}} \hat{x}^{\prime} \\
& -\frac{\sin \gamma}{m} \sqrt{\frac{m^{2}-1+\cos ^{2} \xi \cos ^{2} \gamma}{1-\cos ^{2} \xi \cos ^{2} \gamma}} \hat{y}^{\prime} \\
& +\frac{\sqrt{1-\cos ^{2} \xi \cos ^{2} \gamma}}{m} \hat{z}^{\prime} .
\end{aligned}
$$

The initial polarization vector (before refraction) is given by $\hat{y}$, and has components perpendicular and parallel to the plane of incidence given by $\cos \phi$ and $\sin \phi$, respectively. Therefore, after being refracted into the sample, the polarization vector $\hat{p}$ of the refracted ray is given by:

$$
\hat{p}=(\cos \phi) \hat{p}_{\perp}+(\sin \phi) \hat{p}_{\|} .
$$

(Equation (11) neglects the slightly different transmission coefficients for light polarized parallel and perpendicular to the plane of incidence. This point is treated in detail in the Appendix.)

In the following, it will also be useful to construct a vector which is perpendicular to both $\hat{r}$ and $\hat{p}$, which we will denote by $\hat{p}^{*}$ :

$$
\hat{p}^{*}=-(\sin \phi) \hat{p}_{\perp}+(\cos \phi) \hat{p}_{\|} .
$$

Each of these vectors can be written completely in terms of the angles $\gamma$ and $\xi$ with the help of Equations (4), (9) and (10):

$$
\begin{aligned}
\hat{p}= & \frac{\sin \xi \sin \gamma-\sin \gamma \sin \xi \cos \gamma \cos \xi \frac{\sqrt{m^{2}-1+\cos ^{2} \xi \cos ^{2} \gamma}}{m}}{1-\cos ^{2} \xi \cos ^{2} \gamma} \hat{x}^{\prime} \\
& +\frac{\sin ^{2} \xi \cos \gamma+\sin ^{2} \gamma \cos \xi \frac{\sqrt{m^{2}-1+\cos ^{2} \xi \cos ^{2} \gamma}}{m}}{1-\cos ^{2} \xi \cos ^{2} \gamma} \hat{y}^{\prime} \\
& -\frac{\sin \gamma \cos \xi}{m} \hat{z}^{\prime} .
\end{aligned}
$$

$$
\begin{aligned}
\hat{p}^{*}= & \frac{\sin ^{2} \gamma \cos \xi+\sin ^{2} \xi \cos \gamma \frac{\sqrt{m^{2}-1+\cos ^{2} \xi \cos ^{2} \gamma}}{m}}{1-\cos ^{2} \xi \cos ^{2} \gamma} \hat{x}^{\prime} \\
& +\frac{\sin \gamma \sin \xi \cos \gamma \cos \xi-\sin \xi \sin \gamma \frac{\sqrt{m^{2}-1+\cos ^{2} \xi \cos ^{2} \gamma}}{m}}{1-\cos ^{2} \xi \cos ^{2} \gamma} \hat{y}^{\prime} \\
& +\frac{\sin \xi}{m} \hat{z}^{\prime} .
\end{aligned}
$$

We can now apply these results to find the refraction corrections given by Kamb (1962). As stated above, the conditions for extinction are that $\hat{p}$ is either perpendicular to or parallel to the plane determined by $\hat{r}$ and $\hat{c}$. By considering the situation when $m=1$, it can be shown that the former case corresponds to the " $\mathrm{H}$ " setting ( $c$ axis horizontal) and the latter case corresponds to the "V" setting ( $c$ axis vertical).

For the $\mathrm{H}$ setting, we therefore have the condition $\hat{p} \cdot \hat{c}=0$. For the $\mathrm{V}$ setting, $\hat{p}, \hat{r}$ and $\hat{c}$ are coplanar, and therefore $\hat{p}^{*} \cdot \hat{c}=0$.

For the standard $c$-axis orientation technique, the $c$ axis is initially oriented in the $x-z$ plane. Again using the notation in Kamb (1962), its coordinates in the frame of the section are given by:

$$
\begin{array}{ll}
\hat{c}=-\cos (\xi-\nu) \hat{x}^{\prime}-\sin (\xi-\nu) \hat{z}^{\prime} & \text { H setting } \\
\hat{c}=-\sin (\xi-\mu) \hat{x}^{\prime}+\cos (\xi-\mu) \hat{z}^{\prime} & \text { V setting. }
\end{array}
$$

Briefly, $\nu$ and $\mu$ refer to the angular corrections which must be subtracted from the measured tilt $\xi$ to obtain the true inclination of the $c$ axis with respect to the plane of $(\mathrm{H}$ set- 
ting) or the normal of ( $\mathrm{V}$ setting) the thin section. For a more detailed explanation of the notation, the reader is referred to the original article by Kamb (1962). Applying the two conditions for extinction yields the final results:

$\mathrm{H}$ setting:

$$
\begin{aligned}
& \tan (\xi-\nu) \\
& =\left[\frac{m-\cos \xi \cos \gamma \sqrt{m^{2}-1+\cos ^{2} \xi \cos ^{2} \gamma}}{1-\cos ^{2} \xi \cos ^{2} \gamma}\right] \tan \xi
\end{aligned}
$$

V setting:

$$
\begin{aligned}
& \tan (\xi-\mu) \\
& =\left[\frac{\sin ^{2} \gamma+\sin ^{2} \xi \cos ^{2} \gamma}{m \sin ^{2} \gamma+\sin ^{2} \xi \cos ^{2} \gamma \sqrt{1+\frac{m^{2}-1}{\cos ^{2} \xi \cos ^{2} \gamma}}}\right] \tan \xi .
\end{aligned}
$$

These expressions give numerical results exactly equal to those given in Kamb (1962). (Exact agreement is found after correcting a small algebraic error in Kamb's paper. The denominator in equation (19) in Kamb should read $\lambda^{2}+4 \omega+\sigma^{2}+2 \lambda(2 \omega+\sigma)$.)

\section{Extinction conditions for new technique}

We now proceed to apply the formalism developed in the previous section to calculate the predicted extinction angles as a function of the $c$-axis direction for the four sequences described above for the new technique.

For our purposes, it will be convenient to specify the direction of the $c$ axis using the usual polar $\left(\theta_{c}\right)$ and azimuthal $\left(\phi_{c}\right)$ angles:

$$
\hat{c}=\sin \left(\theta_{c}\right) \cos \left(\phi_{c}\right) \hat{x}^{\prime}+\sin \left(\theta_{c}\right) \sin \left(\phi_{c}\right) \hat{y}^{\prime}+\cos \left(\theta_{c}\right) \hat{z}^{\prime} .
$$

If the sample is initially rotated about the inner axis $\left(\mathrm{A}_{1}\right)$ by some angle $\phi_{\text {in }}$, the $c$ axis is given by Equation (18) with $\phi_{c}$ replaced by $\phi_{c}+\phi_{\text {in }}$ :

$$
\begin{aligned}
& \hat{c}=\sin \left(\theta_{c}\right) \cos \left(\phi_{c}+\phi_{\text {in }}\right) \hat{x}^{\prime} \\
& +\sin \left(\theta_{c}\right) \sin \left(\phi_{c}+\phi_{\text {in }}\right) \hat{y}^{\prime}+\cos \left(\theta_{c}\right) \hat{z}^{\prime}
\end{aligned}
$$

The polarization direction before refraction by the sample, denoted by $\hat{p}_{i}$, is set by the polarizer angle $\phi_{\mathrm{p}}$ :

$$
\hat{p}_{i}=\cos \left(\phi_{\mathrm{p}}\right) \hat{x}+\sin \left(\phi_{\mathrm{p}}\right) \hat{y} .
$$

From this point, the reasoning follows lines very similar to that in the previous subsection. The principal difference here is that the polarization direction prior to refraction, given in Equation (20), has components parallel and perpendicular to the plane of incidence, given by $\cos \left(\phi_{\mathrm{p}}-\phi\right)$ and $\sin \left(\phi_{\mathrm{p}}-\phi\right)$, respectively.

Consequently, the polarization direction in the sample is given by:

$$
\hat{p}=\cos \left(\phi_{\mathrm{p}}-\phi\right) \hat{p}_{\|}+\sin \left(\phi_{\mathrm{p}}-\phi\right) \hat{p}_{\perp} .
$$

As before, we construct the vector $\hat{p}^{*}$ which is perpendicular to both $\hat{r}$ and $\hat{p}$ :

$$
\hat{p}^{*}=-\sin \left(\phi_{\mathrm{p}}-\phi\right) \hat{p}_{\|}+\cos \left(\phi_{\mathrm{p}}-\phi\right) \hat{p}_{\perp} .
$$

The conditions for extinction are again given by the requirement that $\hat{p} \cdot \hat{c}=0$ or $\hat{p}^{*} \cdot \hat{c}=0$. Using Equations (21) and (22), we can write down general equations for the polarizer extinction angle corresponding to these conditions:

$$
\begin{gathered}
\phi_{\mathrm{p}}=\phi+\tan ^{-1}\left[\frac{\hat{p}_{\perp} \cdot \hat{c}}{\hat{p}_{\|} \cdot \hat{c}}\right] \\
\phi_{\mathrm{p}}=\phi+\tan ^{-1}\left[\frac{-\hat{p}_{\|} \cdot \hat{c}}{\hat{p}_{\perp} \cdot \hat{c}}\right],
\end{gathered}
$$

where $\phi, \hat{p}_{\perp}, \hat{p}_{\|}$and $\hat{c}$ are specified by the settings for any particular sequence using Equations (4), (9), (10) and (19). Note that these two conditions will always give extinction angles which differ by $90^{\circ}$. Keeping this fact in mind, we will calculate the extinction angles for a particular sequence using only the first of these two conditions. The second angle will be taken into account explicitly in the analysis of the data, as will be discussed below.

From these results, we can now determine explicit expressions for the predicted extinction angles corresponding to the four specific sequences discussed above.

Sequence 1: $\gamma=0^{\circ}, \xi=0^{\circ}, \phi_{\text {in }}=0^{\circ}$

$$
\phi_{1}=\phi_{c}
$$

Sequence 2: $\gamma=0^{\circ}, \xi=45^{\circ}, \phi_{\text {in }}=0^{\circ}$

$$
\phi_{2}=\tan ^{-1}\left[\frac{\sin \theta_{c} \sin \phi_{c}}{\sin \theta_{c} \cos \phi_{c} \sqrt{1-\frac{\sin ^{2} 45}{m^{2}}}+\frac{\cos \theta_{c} \sin 45}{m}}\right]
$$

Sequence 3: $\gamma=45^{\circ}, \xi=0^{\circ}, \phi_{\text {in }}=0^{\circ}$

$\phi_{3}=-90+\tan ^{-1}\left[-\frac{\sin \theta_{c} \cos \phi_{c}}{\sin \theta_{c} \sin \phi_{c} \sqrt{1-\frac{\sin ^{2} 45}{m^{2}}}-\frac{\cos \theta_{c} \sin 45}{m}}\right]$

Sequence 4: $\gamma=0^{\circ}, \xi=45^{\circ}, \phi_{\text {in }}=-45^{\circ}$

$$
\phi_{4}=\tan ^{-1}\left[\frac{\sin \theta_{c} \sin \left(\phi_{c}-45\right)}{\sin \theta_{c} \cos \left(\phi_{c}-45\right) \sqrt{1-\frac{\sin ^{2} 45}{m^{2}}+\frac{\cos \theta_{c} \sin 45}{m}}}\right] .
$$

There are several small corrections to these results which are discussed in detail in the Appendix.

\section{EXPERIMENT}

\section{Apparatus}

A sketch of the experimental apparatus is shown in Figure 4. The essential components are rotation stages for the polarizers as well as for the axes of the universal stage. The lubrication for the stages was replaced with grease suitable for cold-room temperatures. The imaging was performed with a charge coupled device (CCD) camera connected to a "SNAPPY" frame grabber board attached to the parallel port of the computer. The light source was a fiber-optic backlight illuminator. The components were mounted on an optical breadboard for ease of alignment. Before any imaging was performed, all of the components were aligned using a diode laser as follows: Rotation stages 1 and 2 were adjusted so that the laser-beam reflection from the polarizers overlapped the incident beam, insuring that each polarizer was perpendicular to the light path. A glass substrate was placed in stage 5 , and the universal stage was adjusted so that the 


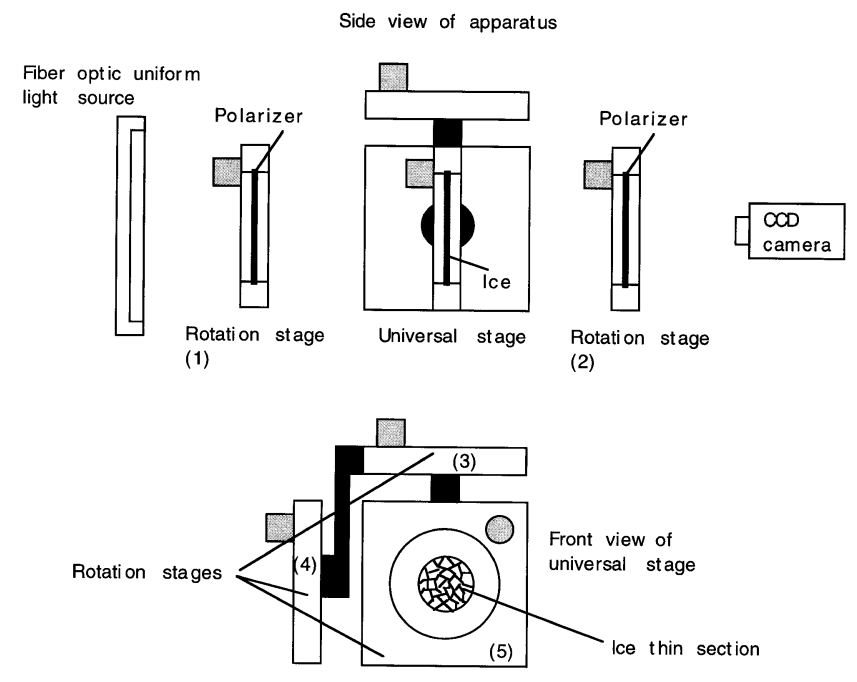

Fig. 4. Experimental set-up. All components mount to a standard optical bench.

substrate was perpendicular to the light path when the angles of stages 3 and 4 were set to $0^{\circ}$. Then stage 3 was rotated to Brewster's angle for glass, and the absolute direction of polarization for polarizer 1 was set by finding extinction of the laser beam upon reflection from the glass. Once this was found, polarizer 1 was rotated by $90^{\circ}$ so that its polarization direction was along the $y$ axis. The glass substrate was then removed, and polarizer 2 was adjusted to the angle which gave the best extinction.

\section{Measurements}

The experimental procedure is divided naturally into two parts: the capturing of the images, and the subsequent analysis to determine $c$-axis orientations.

The apparatus was installed at the U.S. National Ice Core Laboratory (NICL), Denver, Colorado, where measurements were performed on an ice thin section from Taylor Dome. The thin section was roughly $30 \mathrm{~mm}$ long by $15 \mathrm{~mm}$ wide by $0.4 \mathrm{~mm}$ thick. (Modifications for $100 \mathrm{~mm}$ diameter sections are in progress.) The average size of the ice grains was approximately $2 \mathrm{~mm}$. The PG-compatible computer used to grab the images was placed in a hot box next to the apparatus, with a cable running to the CCD camera. All of the other components, including the fiber-optic light-source power supply and lamp were placed directly in the cold room, where the temperature was approximately $-20^{\circ} \mathrm{C}$.

The four sequences of images described above were captured. Figure 5 shows one image $\left(\right.$ taken at $0^{\circ}$ ) from each of the four sequences. The image size of $320 \times 240$ pixels was sufficient to resolve all of the grains $(>70)$ in the section. The memory required to store the 84 gray-scale images from all four sequences was approximately 6.5 MB. Once the images were stored, all the analysis required to determine the fabric was performed outside the cold room.

\section{Results and analysis}

In this section, I describe the specific procedure used to determine the $c$-axis directions of the ice grains from the image data. Since the analysis is the same for every grain in the sample, the procedure is discussed referring to one particular grain (grain 4 in Fig. 5).

The intensity of a particular grain is measured by draw- ing a rectangular window inside the grain, and averaging the gray-scale values for the pixels in that window. The same window is used for all the images in one given sequence. Figure 6 shows a plot of the intensity of light measured for grain 4 as a function of the polarizer angle for each sequence. Theoretically, the intensity variations should be sinusoidal. However, the camera used had a very non-linear intensity response, which was discovered later by checking it with a gray-scale calibration slide. Nevertheless, with a parabolic fit to the three lowest intensities (i.e. highest gray scale), the extinction angle (for each sequence) can typically be determined to within about $1^{\circ}$. (When the $c$ axis happens to be closely aligned with the refracted ray for a particular sequence, the intensity curve can have low contrast, and the error in the extinction angle will be high. In this case, the extinction angles from the remaining sequences are used to determine the $c$-axis direction.) The goal is then to determine the unique polar and azimuthal angles specifying the $c$-axis direction, denoted by $\left(\theta_{c}, \phi_{c}\right)$, which are consistent with the measured set of extinction angles. To do this, we construct the following function of $\theta_{c}$ and $\phi_{c}$ :

$$
\begin{aligned}
R^{2}\left(\theta_{c}, \phi_{c}\right) & =\min \left[\left(\phi_{1}-\phi_{1}^{\exp }\right)^{2},\left(\phi_{1}-\phi_{1}^{\exp }-90\right)^{2}\right] \\
& +\min \left[\left(\phi_{2}-\phi_{2}^{\exp }\right)^{2},\left(\phi_{2}-\phi_{2}^{\exp }-90\right)^{2}\right] \\
& +\min \left[\left(\phi_{3}-\phi_{3}^{\exp }\right)^{2},\left(\phi_{3}-\phi_{3}^{\exp }-90\right)^{2}\right] \\
& +\min \left[\left(\phi_{4}-\phi_{4}^{\exp }\right)^{2},\left(\phi_{4}-\phi_{4}^{\exp }-90\right)^{2}\right],
\end{aligned}
$$

where the set of extinction angles $\left(\phi_{1}, \phi_{2}, \phi_{3}, \phi_{4}\right)$ are functions of $\theta_{c}$ and $\phi_{c}$ as determined by Equations (25-28), and $\left(\phi_{1}^{\exp }, \phi_{2}^{\exp }, \phi_{3}^{\exp }, \phi_{4}^{\exp }\right)$ are the experimental results for the extinction angles. Choosing the minimum of $\left(\phi_{n}-\phi_{n}^{\exp }\right)^{2}$ and $\left(\phi_{n}+90-\phi_{n}^{\exp }\right)^{2}$ accounts for the $90^{\circ}$ degeneracy in the extinction angles as discussed in the theory section. Since we are implicitly requiring that the range for the azimuthal angle be restricted to $0^{\circ} \leq \phi_{c} \leq 180^{\circ}$, we allow the polar angle to span the values $-90^{\circ} \leq \theta_{c} \leq 90^{\circ}$ in order to cover the full range of solid angles for the $c$-axis orientations. (For the tabulated results, the angles were converted back to the usual ranges.) The values of $\left(\theta_{c}, \phi_{c}\right)$ which minimize $R^{2}$ determine the direction of the $c$ axis. It is not necessary to search over all possible values of $\theta_{c}$ and $\phi_{c}$. Since $\phi_{1}^{\exp }$ immediately determines $\phi_{c}$ up to $90^{\circ}$, we search for a minimum in $R^{2}$ over the restricted range of values $\left(-90^{\circ} \leq \theta_{c} \leq 90^{\circ}, \phi_{c}=\phi_{1}^{\exp }\right)$ and $\left(-90^{\circ} \leq \theta_{c} \leq 90^{\circ}, \phi_{c}=\phi_{1}^{\exp }+90^{\circ}\right)$.

In Figure 7, a plot is shown of the functions $R^{2}\left(\theta_{c}, \phi_{c}=\phi_{1}\right)$ and $R^{2}\left(\theta_{c}, \phi_{c}=\phi_{1}+90\right)$ vs $\theta_{c}$ for the results from grain 4 . From this plot, we can read off the angles which minimize $R^{2}$ to be $\phi_{c}=67.1, \theta_{c}=-76.2$. Once the minimum in $R^{2}$ is determined for a particular $\left(\theta_{c}, \phi_{c}\right)$, we then relax the condition that $\phi_{c}$ be exactly equal to $\phi_{1}^{\exp }$ up to $90^{\circ}$. A slightly lower $R^{2}$ is obtained by letting $\phi_{c}$ vary. The minimum $R^{2}$ values are all $<3$, which implies that agreement between the measured and theoretical extinction angles is typically better than $1^{\circ}$.

With this procedure, the $c$-axis direction was found for 15 of the grains in the sample, chosen randomly. Due to time constraints, measurements were not able to be completed using the standard $c$-axis technique during the visit to NICL. Instead, the ice was shipped out to the Byrd Polar Research Center, Columbus, Ohio, a few months later, and measurements were performed there. During the course of an afternoon, the $c$-axis orientation of the same 15 grains was determined.

The results are shown in Table 1 . For the standard tech- 


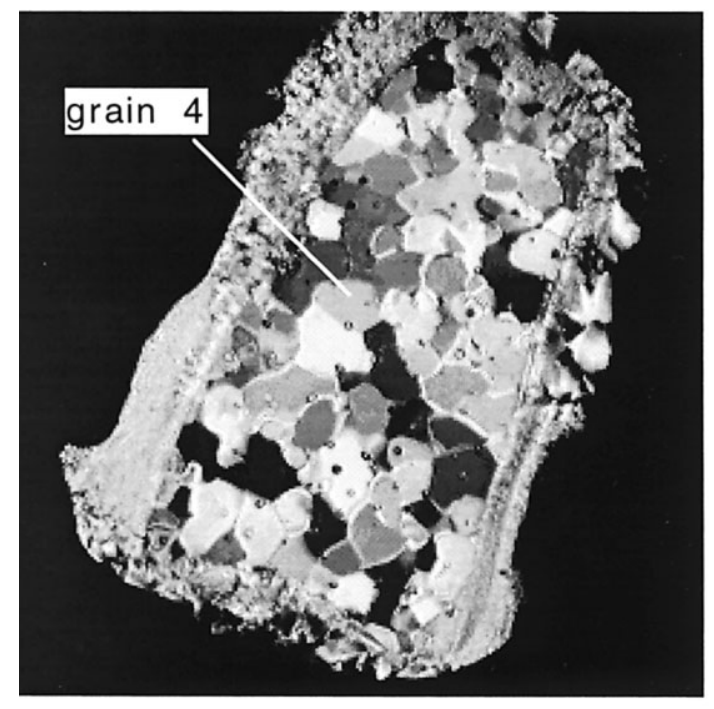

sequence 1

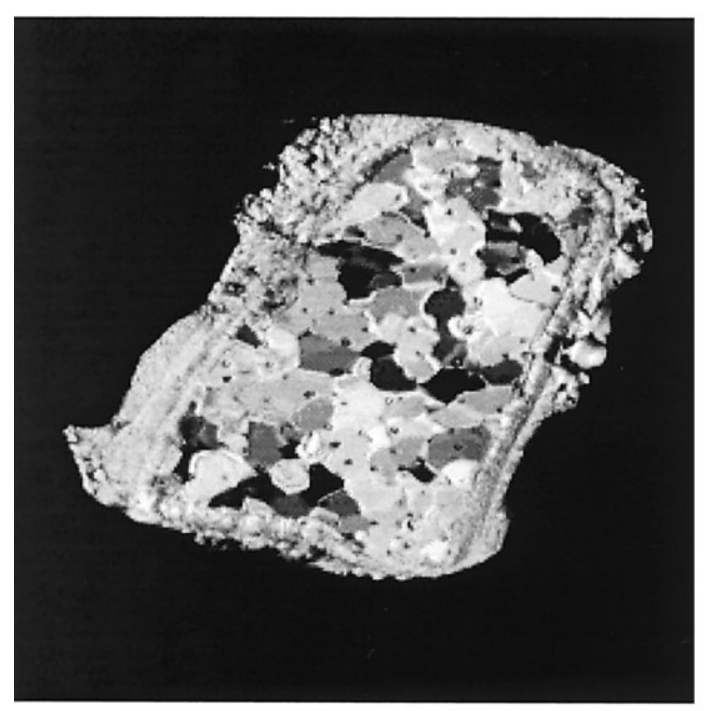

sequence 3

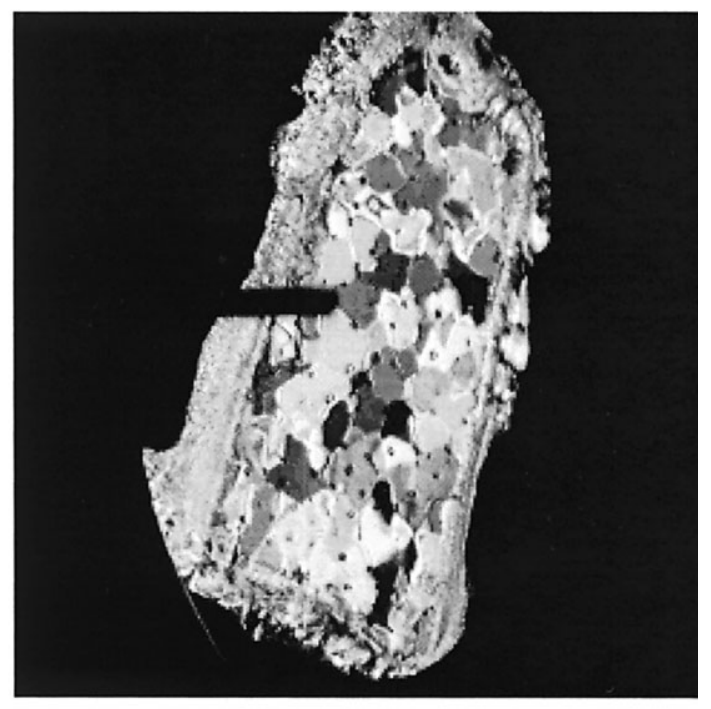

sequence 2

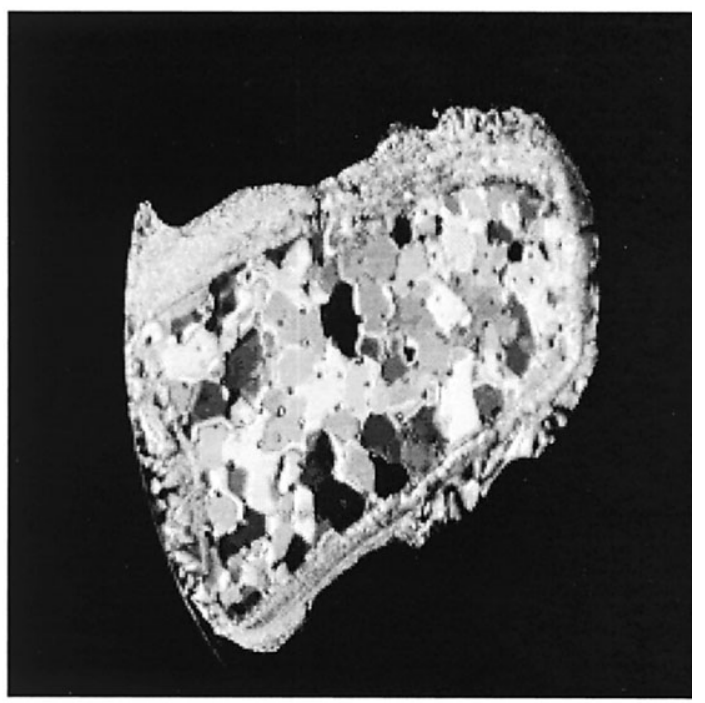

sequence 4

Fig. 5. Images of ice sample for each of the four sequences. Polarizers are set to $0^{\circ}$ for these images. The rectangular black area in sequence 2 is due to a small post on the rotation stage which cast a shadow on the sample for this orientation.

nique measurements, the sample was not mounted in the rotation stage with the same azimuthal orientation as it had

Table 1 . The polar and azimuthal angles $\left({ }^{\circ}\right)$ of 15 grains

\begin{tabular}{rcrccc}
\hline Grain No. & $\begin{array}{c}\theta \text { (standard } \\
\text { technique) }\end{array}$ & $\begin{array}{c}\phi(\text { standard } \\
\text { technique) }\end{array}$ & $\begin{array}{c}\theta \text { (new } \\
\text { technique) }\end{array}$ & $\begin{array}{c}\phi(\text { new } \\
\text { technique) }\end{array}$ & $\begin{array}{c}\text { minimum } \\
R^{2}\end{array}$ \\
\hline 1 & 51 & 204 & 49.2 & 205.5 & 2.22 \\
2 & 87 & 149 & 84.9 & 149.4 & 0.72 \\
3 & 37 & 44 & 37.7 & 44.8 & 0.035 \\
4 & 74 & 248 & 76.0 & 247.2 & 2.48 \\
5 & 51 & 35 & 51.7 & 34.8 & 1.16 \\
6 & 45 & 309 & 45.4 & 308.5 & 1.65 \\
7 & 47 & 166 & 46.4 & 166.8 & 0.48 \\
8 & 37 & 238 & 37.3 & 239.7 & 0.83 \\
9 & 39 & 331 & 37.0 & 332.4 & 0.47 \\
10 & 37 & 63 & 38.0 & 60.1 & 2.25 \\
11 & 87 & 75 & 87.0 & 74.6 & 1.70 \\
12 & 57 & 16 & 57.9 & 16.7 & 2.06 \\
13 & 35 & 30 & 34.2 & 27.3 & 0.66 \\
14 & 54 & 50 & 54.4 & 48.0 & 2.66 \\
15 & 26 & 105 & 27.9 & 107.2 & 0.83 \\
& & & 46.8 & 197.9 & 0.33 \\
& & & & &
\end{tabular}

been for the previous image measurements. Consequently, for comparison purposes, the azimuthal angles for the standard technique have all been adjusted by a uniform additive constant.

\section{DISGUSSION}

Good agreement is achieved between the results from the two different measurement techniques. The error in the measurements using the standard technique is approximately $2^{\circ}$ for the polar and azimuthal angles. For the new technique, the error is estimated at approximately $1-2^{\circ}$ for both of the angles. It is likely that the manual setting of the polarizers was responsible for most of this error, and it is expected that an accuracy of better than $0.5^{\circ}$ for both angles can be achieved with a fully automated system. Note that in one particular case (grain 15) two $c$-axis directions were found consistent with the measured extinction angles. This has led to the realization that the four particular sequences chosen were not generic enough to uniquely determine every possible $c$-axis direction. A check has been made that 
Sequence 1, grain 4

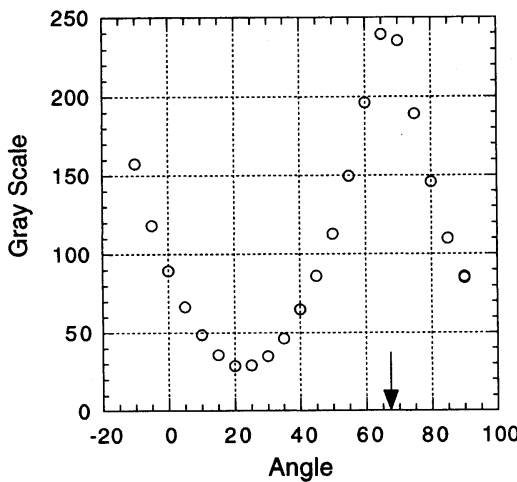

Sequence 3 , grain 4

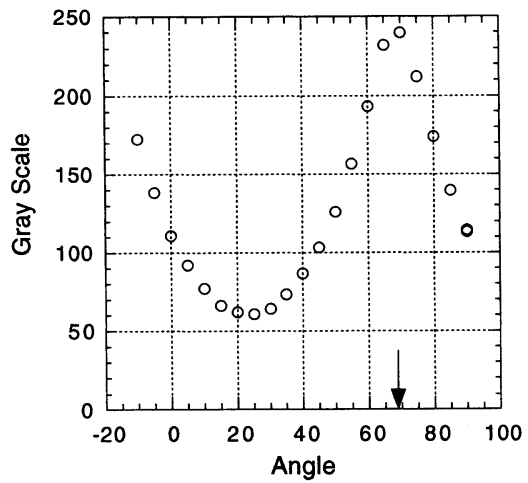

Sequence 2, grain 4

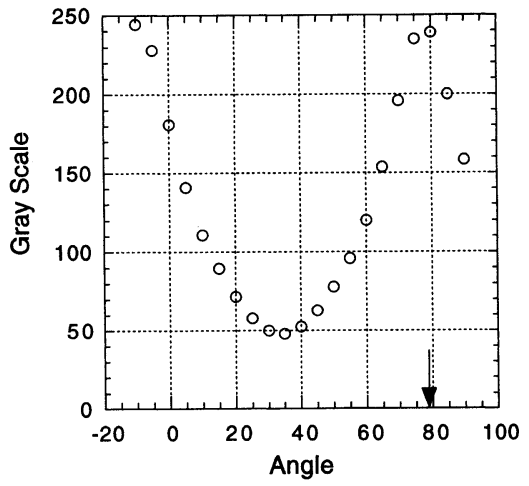

Sequence 4, grain 4

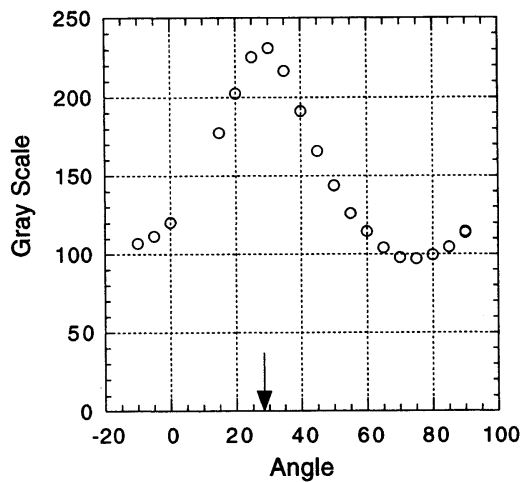

Fig. 6. Plots of gray scale $(255=$ black, $0=$ white) vs angle for grain 4 for each of the four sequences. The angle of extinction is indicated by the arrow. In sequence 4 , data for two angles $\left(5^{\circ}, 10^{\circ}\right)$ are missing due to corrupted image files. This did not affect the results since in the few cases where the extinction angle from sequence 4 could not be determined, data from the other three were sufficient to uniquely determine the $c$ axis.

this can be corrected by adding a fifth sequence, and possibly by choosing a different (less symmetric) set of four.

\section{$R^{2}$ grain 4}

Circles: $\phi_{c}=67.1^{\circ}$

Squares: $\phi_{c}=67 \cdot 1^{\circ}+90^{\circ}=157.1^{\circ}$

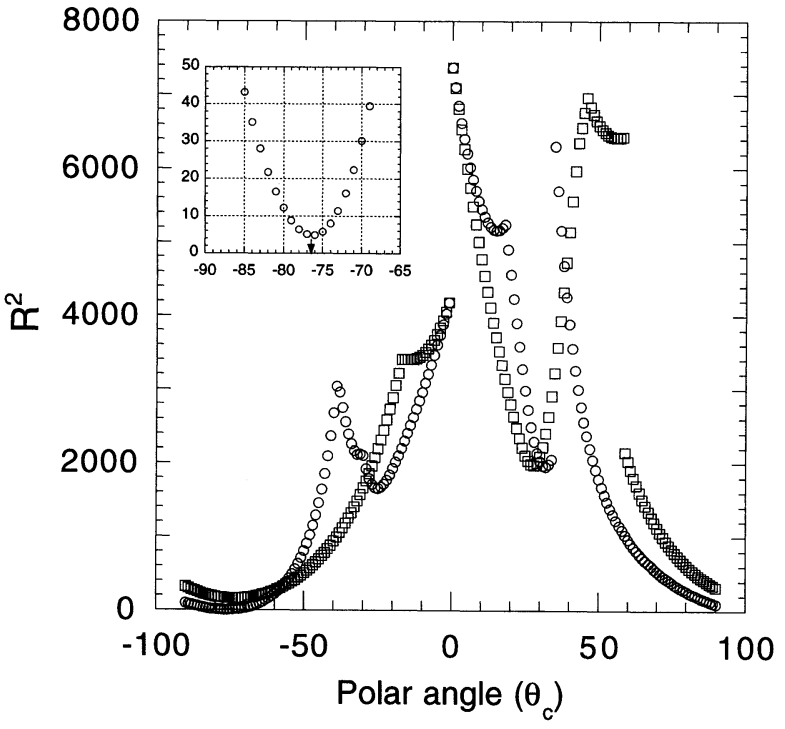

Fig. 7. Plot of $R^{2}$ vs $\theta_{c}$ for $\phi_{c}=67.1^{\circ}$, and $\phi_{c}=157.1^{\circ}$. The inset shows a blow-up of the region around the minimum of $R^{2}$ (indicated by the arrow) which occurs at $\theta_{c}=-76.3^{\circ}, \phi_{c}=67.1^{\circ}$. The true minimum of $R^{2}$ (allowing $\phi_{c}$ to vary) is then found to be at $\theta_{c}=-76.0^{\circ}$, $\phi_{c}=67.2^{\circ}$. Converting these to the conventional ranges gives $\theta_{c}=76.0^{\circ}, \phi_{c}=247.2^{\circ}$.
The azimuthal angles for the analyzed grains ranged from $0^{\circ}$ to $360^{\circ}$ while the span of the polar angles was about $25-$ $90^{\circ}$. The same experiment described here was also performed for a thin section of randomly oriented quartz grains. For this quartz sample, the polar angles of the analyzed grains ranged down to $3^{\circ}$ and there was no difficulty in analyzing the grains with small polar angles.

\section{Automation of technique}

Although the measurements described here were taken and analyzed manually, the development of a fully automated version of the apparatus is almost complete. The data-taking has been automated by the addition of motorized rotation stages, along with software which integrates the image-grabbing and rotation-stage control. The new rotation stages are large enough to accommodate sections $100 \mathrm{~mm}$ in diameter. The original CCD camera has been replaced with one which has a highly linear response. Tests have shown that it takes $<10$ min to completely image one thin section. Work on automating the analysis part of the technique is at an advanced stage. Currently, the position of each grain must be specified in each of the sequences by hand (by drawing a window in the image), but a program which maps the position of a grain in the first sequence to the correct position in all the others is almost complete. Once the position of a grain in a given sequence is specified, it takes $<1 \mathrm{~s}$ to calculate the extinction angle from the image data. Using the four extinction angles, it takes only a few seconds to calculate the $c$-axis direction using the procedure described above. Detailed results from the automated set-up will be reported elsewhere. 


\section{CONGLUSIONS}

I have described the theoretical and experimental basis of a new technique for ice-fabric analysis. The technique relies on a set of digital images taken of an ice thin section viewed at different angles through crossed polarizers. The technique lends itself to a completely automated process, and plans for automation are well underway. A big advantage of the method is that the data for a large number of samples can be imaged in a fairly short time. Once the images are acquired, the burden of analysis is shifted from laborious manual manipulations inside the cold room to computer calculations outside the cold room. The results from an automated analysis are expected to be more accurate than the old technique, and highly reproducible.

It is expected that the increased statistics on ice fabrics will lead to many advances in our understanding of anisotropic flow laws for ice, active processes such as polygonization and recrystallization, and more.

Upon completion of this paper, I became aware that another group (Wang and Azuma, 1999) had recently reported work on a technique for fabric analysis using a technique conceptually similar, although substantially different in detail, to the one described herein.

\section{ACKNOWLEDGEMENTS}

I thank R. Alley and J. Fitzpatrick for their support, advice and encouragement in this endeavor. The NICL provided the facilities for the image measurements. J. Fitzpatrick made the ice thin section used, and provided invaluable help with the set-up of the experiment. I would like to thank L. Thompson and E. Mosley-Thompson for allowing me to use the cold room at the Byrd Polar Research Center for the standard $c$ axis measurements. I also thank N. Azuma, D. Hansen, T. H. Jacka and D. Russell-Head for many helpful suggestions. This work was supported by Ohio University Research and Sponsored Programs.

\section{REFERENCES}

Alley, R. B. 1992. Flow-law hypotheses for ice-sheet modeling. F. Glaciol., 38(129), 245-256.

Alley, R. B., A. J. Gow, S. J. Johnsen, J. Kipfstuhl, D. A. Meese and Th. Thorsteinsson. 1995. Comparison of deep ice cores. Nature, 373(6513), 393-394.

Alley, R. B., A. J. Gow, D. A. Meese, J. J. Fitzpatrick, E. D. Waddington and J. F. Bolzan. 1997. Grain-scale processes, folding and stratigraphic disturbance in the GISP2 ice core. 7. Geophys. Res., 102(C12), 26,819-26,830.

Anandakrishnan, S., J.J. Fitzpatrick, R. B. Alley, A. J. Gow and D. A. Meese. 1994. Shear-wave detection of asymmetric c-axis fabrics in the GISP2 ice core, Greenland. 7. Glaciol., 40(136), 491-496.

Azuma, N. and A. Higashi. 1985. Formation processes of ice fabric pattern in ice sheets. Ann. Glaciol., 6, 130-134.

Bentley, C. R. 1971. Seismic anisotropy in the West Antarctic ice sheet. In Grary, A.P., ed. Antarctic snow and ice studies II. Washington, DC, American Geophysical Union, 131-177. (Antarctic Research Series 16.)

Berek, M. 1923. NeueWege zur Universalmethode. Neues Jahrb. Mineral. Geol. Paläontol., 38, 34-62.

Blankenship, D. D. and C. R. Bentley. 1987. The crystalline fabric of polar ice sheets inferred from seismic anisotropy. International Association of Hydrological Sciences Publication 170 (Symposium at Vancouver $1987-$ The Physical Basis of Ice Sheet Modelling), 17-28.

Budd, W. F. and T. H. Jacka. 1989. A review of ice rheology for ice sheet modelling. Cold Reg. Sci. Technol., 16(2), 107-144.

Fueten, F. 1997. A computer-controlled rotating polarizer stage for the petrographic microscope. Comput. Geosci., 23 (2), 203-208.

Grootes, P. M., M. Stuiver, J.W. C. White, S. Johnsen and J. Jouzel. 1993. Comparison of oxygen isotope records from the GISP2 and GRIP
Greenland ice cores. Nature, 366(6455), 552-554.

Herron, S. L., C. C. Langway, Jr and K. A. Brugger. 1985. Ultrasonic velocities and crystalline anisotropy in the ice core from Dye 3, Greenland. In Langway, C. C., Jr, H. Oeschger and W. Dansgaard, eds. Greenland ice core: geophysics, geochemistry, and the environment. Washington, DC, American Geophysical Union, 23-31. (Geophysical Monograph 33.)

Kamb, W. B. 1958. Isogyres in interference figures. Am. Mineral., 43, 1029-1067.

Kamb, W. B. 1962. Refraction corrections for universal stage measurements. I. Uniaxial crystals. Am. Mineral., 47(3), 227-245.

Kohnen, H. and A. J. Gow. 1979. Ultrasonic velocity investigations of crystal anisotropy in deep ice cores from Antarctica. 7. Geophys. Res., 84(C8), 4865-4874.

Lange, M. A. 1988. A computer-controlled system for ice-fabric analysis on a Rigsby stage. Ann. Glaciol., 10, 92-94.

Langway, C. C., Jr. 1958. Ice fabrics and the universal stage. SIPRE Tech. Rep. 62.

Lipenkov, V.Ya., N. I. Barkov, P. Duval and P. Pimienta. 1989. Crystalline texture of the $2083 \mathrm{~m}$ ice core at Vostok Station, Antarctica. F. Glaciol., 35(121), 392-398.

Liu, C., C. R. Bentley and N. E. Lord. 1994. $c$ axes from radar depolarization experiments at Upstream B Camp, Antarctica, in 1991-92. Ann. Glaciol., 20, 169-176.

MacAyeal, D. R., R. A. Bindschadler and T. A. Scambos. 1995. Basal friction of Ice Stream E, West Antarctica. 7. Glaciol., 41(138), 247-262.

Morgan, V. I., E. R. Davis and E. Wehrle. 1984. A Rigsby stage with remote computer compatible output. Cold Reg. Sci. Technol., 10(1), 89-92.

Mori, Y., T. Hondoh and A. Higashi. 1985. Development of an automatic ice fabric analyser. Ann. Glaciol., 6, 281-283.

Rigsby, G. P. 1951. Grystal fabric studies on Emmons Glacier, Mount Rainier, Washington. f. Geol., 59(6), 590-598.

Taylor, K. C. and 9 others. 1993. Electrical conductivity measurements from the GISP2 and GRIP Greenland ice cores. Nature, 366 (6455), 549-552.

Wang Yun and N. Azuma. 1999. A new automatic ice-fabric analyzer which uses image-analysis techniques. Ann. Glaciol., 29, 155-162.

Zagorodnov, V. S., J. J. Kelley, L. Thompson and O. Watanabe. 1994. Continuous study of an ice core: ECM, fine stratigraphy, air bubbles and crystals. Natl. Inst. Polar Res. Mem., Special Issue 49, 281-290.

\section{APPENDIX}

\section{CORREGTIONS FOR SYSTEMATIG ERRORS}

\section{Correction for off-axis grains}

In the standard $c$-axis analysis technique, the person moves the eye so that it is always directly over the grain being analyzed. In other words, the light always travels parallel to the $z$ axis outside of the section. In contrast, for the technique described here, the camera which images the thin section sits in a fixed position. Except for grains located close to the center of the section, the light which passes through a particular grain and is imaged follows a path which deviates from the $z$ axis outside the section. The situation is shown in Figure 8.

There are two consequences of this light-path deviation which must be taken into account. The first is that there are corrections to the tilt of the sample with respect to the direction of propagation of the light. The second is that the initial

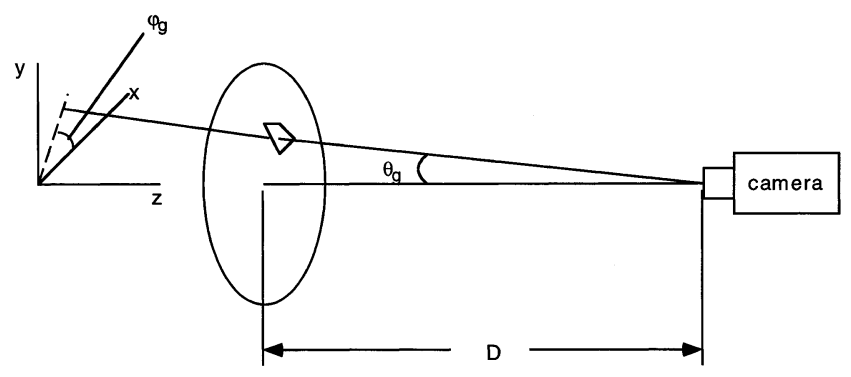

Fig. 8. Diagram of path of light passing through an off-axis grain. $\phi_{\mathrm{g}}$ is the azimuthal angle of the projection of the grain's position onto the $x-y$ plane. $\theta_{\mathrm{g}}$ is the angle between the light path and the $z$ axis. 
polarization of the light must be corrected because the light from the source passes through the polarizer at an angle different from $90^{\circ}$. Each of these corrections is straightforward to implement.

\section{Tilt correction}

Let us define two angles as shown in Figure 8. $\theta_{\mathrm{g}}$ specifies the angle between the $z$ axis and the line between the grain and the camera lens. $\phi_{\mathrm{g}}$ specifies the azimuthal angle of the grain in the laboratory coordinate system. Suppose that, before any rotations, the location of the grain in the laboratory frame is specified by the displacement vector $a \hat{x}+b \hat{y}$. After a rotation of the section by the rotation angles , $\phi_{\text {in }}, \gamma$ and $\xi$, the new position coordinates of the grain in the laboratory frame will be given by:

$$
\left(\begin{array}{l}
a^{\prime} \\
b^{\prime} \\
c^{\prime}
\end{array}\right)=M\left(\begin{array}{ccc}
\cos \left(\phi_{\text {in }}\right) & -\sin \left(\phi_{\text {in }}\right) & 0 \\
\sin \left(\phi_{\text {in }}\right) & \cos \left(\phi_{\text {in }}\right) & 0 \\
0 & 0 & 1
\end{array}\right)\left(\begin{array}{l}
a \\
b \\
0
\end{array}\right),
$$

where $M$ is the rotation matrix defined in Equation (2).

The angles $\theta_{\mathrm{g}}$ and $\phi_{\mathrm{g}}$ can then be calculated in terms of these coordinates and the distance $D$ between the camera and the center of the section, as follows:

$$
\begin{aligned}
& \theta_{\mathrm{g}}=\tan ^{-1}\left(\frac{\sqrt{a^{\prime 2}+b^{\prime 2}}}{D-c^{\prime}}\right) \\
& \phi_{\mathrm{g}}=\tan ^{-1}\left(\frac{b^{\prime}}{a^{\prime}}\right) .
\end{aligned}
$$

The extra tilt of the grain is accounted for as follows: We construct the rotation matrix, denoted by $M_{\text {light path }}$ which rotates the section by the angle $\theta_{\mathrm{g}}$ about an axis perpendicular to both the vector $a^{\prime} \hat{x}+b^{\prime} \hat{y}$ and the $z$ axis:

$$
\begin{aligned}
M_{\text {light path }} & =\left(\begin{array}{ccc}
\cos \phi_{\mathrm{g}} & -\sin \phi_{\mathrm{g}} & 0 \\
\sin \phi_{\mathrm{g}} & \cos \phi_{\mathrm{g}} & 0 \\
0 & 0 & 1
\end{array}\right)\left(\begin{array}{ccc}
\cos \theta_{\mathrm{g}} & 0 & \sin \theta_{\mathrm{g}} \\
0 & 1 & 0 \\
-\sin \theta_{\mathrm{g}} & 0 & \cos \theta_{\mathrm{g}}
\end{array}\right)\left(\begin{array}{ccc}
\cos \phi_{\mathrm{g}} & \sin \phi_{\mathrm{g}} & 0 \\
-\sin \phi_{\mathrm{g}} & \cos \phi_{\mathrm{g}} & 0 \\
0 & 0 & 1
\end{array}\right) \\
& =\left(\begin{array}{ccc}
\cos \theta_{\mathrm{g}} \cos ^{2} \phi_{\mathrm{g}}+\sin ^{2} \phi_{\mathrm{g}} & \cos \phi_{\mathrm{g}} \sin \phi_{\mathrm{g}}\left(\cos \theta_{\mathrm{g}}-1\right) & \sin \theta_{\mathrm{g}} \cos \phi_{\mathrm{g}} \\
\cos \phi_{\mathrm{g}} \sin \phi_{\mathrm{g}}\left(\cos \theta_{\mathrm{g}}-1\right) & \cos \theta_{\mathrm{g}} \sin ^{2} \phi_{\mathrm{g}}+\cos ^{2} \phi_{\mathrm{g}} & \sin \theta_{\mathrm{g}} \sin \phi_{\mathrm{g}} \\
-\sin \theta_{\mathrm{g}} \cos \phi_{\mathrm{g}} & -\sin \theta_{\mathrm{g}} \sin \phi_{\mathrm{g}} & \cos \theta_{\mathrm{g}}
\end{array}\right)
\end{aligned}
$$

The full rotation of a grain for a given sequence is then given by the combined rotation matrix $M_{\text {combined }}=M_{\text {light path }} M$. We can now generalize the results of the theory section using the rotation matrix $M_{\text {combined }}$ in place of $M$ throughout. Without providing the explicit steps, the results are summarized below. Let $M_{\text {combined }}(i, j)$ refer to the $i, j$ element of the matrix, where $i$ and $j$ index the row and column, respectively. Applying $M_{\text {combined }}$ to $\hat{z}$ determines $\hat{z}^{\prime}$ in terms of the unprimed coordinates:

$$
\hat{z}^{\prime}=M_{\text {combined }}(1,3) \hat{x}+M_{\text {combined }}(2,3) \hat{y}+M_{\text {combined }}(3,3) \hat{z} \text {. }
$$

The azimuthal angle $\phi$ is given by:

$$
\tan \phi=\frac{M_{\text {combined }}(2,3)}{M_{\text {combined }}(1,3)} .
$$

The inverse of $M_{\text {combined }}$ is given by:

$$
M_{\text {combined }}^{-1}=M^{T} M_{\text {light path }}^{T},
$$

where $T$ refers to the transpose of the matrix.
Applying $M_{\text {combined }}^{-1}$ to $\hat{z}^{\prime}$ determines $\hat{z}$ in terms of the primed coordinates:

$\hat{z}=M_{\text {combined }}^{-1}(1,3) \hat{x}^{\prime}+M_{\text {combined }}^{-1}(2,3) \hat{y}^{\prime}+M_{\text {combined }}^{-1}(3,3) \hat{z}^{\prime}$.

The vector $\hat{r}$ is given by:

$$
\begin{aligned}
\hat{r} & =\frac{M_{\text {combined }}^{-1}(1,3)}{m} \hat{x}^{\prime}+\frac{M_{\text {combined }}^{-1}(2,3)}{m} \hat{y}^{\prime} \\
& +\sqrt{1-\left[\frac{M_{\text {combined }}^{-1}(1,3)}{m}\right]^{2}-\left[\frac{M_{\text {combined }}^{-1}(2,3)}{m}\right]^{2}} \hat{z}^{\prime} .
\end{aligned}
$$

Defining $\hat{r}_{x^{\prime}}, \hat{r}_{y^{\prime}}$ and $\hat{r}_{z^{\prime}}$ to correspond to the $x^{\prime}, y^{\prime}$ and $z^{\prime}$ components of $\hat{r}$, as expressed above, we can also immediately write down expressions for $\hat{p}_{\perp}$ and $\hat{p}_{\|}$:

$$
\begin{gathered}
\hat{p}_{\perp}=\frac{\hat{r}_{y^{\prime}}}{\sqrt{\hat{r}_{x^{\prime}}^{2}+r_{y^{\prime}}^{2}}} \hat{x}^{\prime}-\frac{\hat{r}_{x^{\prime}}}{\sqrt{\hat{r}_{x^{\prime}}^{2}+\hat{r}_{y^{\prime}}^{2}}} \hat{y}^{\prime} \\
\hat{p}_{\|}=-\frac{\hat{r}_{x^{\prime}} \hat{r}_{z^{\prime}}}{\sqrt{\hat{r}_{x^{\prime}}^{2}+\hat{r}_{y^{\prime}}^{2}}} \hat{x}^{\prime}-\frac{\hat{r}_{y^{\prime}} \hat{r}_{z^{\prime}}}{\sqrt{\hat{r}_{x^{\prime}}^{2}+\hat{r}_{y^{\prime}}^{2}}} \hat{y}^{\prime}+\sqrt{\hat{r}_{x^{\prime}}^{2}+\hat{r}_{y^{\prime}}^{2}} z^{\prime} .
\end{gathered}
$$

Substituting the above expressions for $\hat{p}_{\perp}, \hat{p}_{\|}$and $\phi$ in Equations (23) and (24) yields the extinction angle fully corrected for the extra tilt of off-axis grains.

\section{Off-axis polarization correction}

For off-axis grains, the light from the source passes through the polarizer at an angle of $\theta_{\mathrm{g}}$. Consider the projection of the grain onto the $x-y$ plane.

The component of the polarization along the direction of the grain projection is unchanged, while the component perpendicular to the projection is decreased by $\cos \theta_{\mathrm{g}}$. Thus the corrected angle of polarization $\phi_{\mathrm{p}}^{\prime}$ is related to the actual polarizer angle $\phi_{\mathrm{p}}$ by the equation:

$$
\phi_{\mathrm{p}}^{\prime}=\phi_{\mathrm{g}}+\tan ^{-1}\left[\frac{\tan \left(\phi_{\mathrm{p}}-\phi_{\mathrm{g}}\right)}{\cos \left(\theta_{\mathrm{g}}\right)}\right] \text {. }
$$

The experimentally measured angles of extinction must therefore be adjusted by adding the correction $\triangle \phi_{\mathrm{p}}$ (light path):

$$
\triangle \phi_{\mathrm{p}}(\text { light path })=\tan ^{-1}\left[\frac{\tan \left(\phi_{\mathrm{p}}-\phi_{\mathrm{g}}\right)}{\cos \left(\theta_{\mathrm{g}}\right)}\right]-\left(\phi_{\mathrm{p}}-\phi_{\mathrm{g}}\right) \text {. }
$$

\section{Correction for light-intensity variations}

The angle of extinction for a given sequence is found by fitting the data to find the angle which minimizes the transmitted light through a grain. The determination of this minimum will be influenced by any variations in the light intensity as a function of polarizer angle not due to the birefringence of the ice. Corrections are therefore required to account for variations in the light intensity due to the difference in transmission for light polarized perpendicular and parallel to the plane of incidence. These corrections are most easily applied directly to the experimental measurements for intensity. Con- 
sider the Fresnel equations for transmission of light across two media with differing index of refraction:

$$
T_{\perp}=\frac{4\left(\frac{n_{1}}{n_{2}}\right) \cos \theta_{\mathrm{i}} \cos \theta_{\mathrm{t}}}{\left[\left(\frac{n_{1}}{n_{2}}\right) \cos \theta_{\mathrm{i}}+\cos \theta_{\mathrm{t}}\right]^{2}} \quad T_{\|}=\frac{4\left(\frac{n_{1}}{n_{2}}\right) \cos \theta_{\mathrm{i}} \cos \theta_{\mathrm{t}}}{\left[\cos \theta_{\mathrm{i}}\left(\frac{n_{1}}{n_{2}}\right) \cos \theta_{\mathrm{t}}\right]^{2}} .
$$

$T_{\perp}$ and $T_{\|}$are the transmitted intensities for light polarized perpendicular and parallel to the plane of incidence, respectively. $\theta_{\mathrm{i}}$ and $\theta_{\mathrm{t}}$ are the incident and transmitted beam angles with respect to the normal to the interface, and $n_{1}$ and $n_{2}$ are the indices of refraction in the first and second media.

Equation (Al3) can be applied repetitively to each of the interfaces in the system (air/ice, ice/glass, glass/air) to find the overall intensity of transmitted light for light polarized perpendicular and parallel to the plane of incidence, which we denote by $T_{\perp}^{\text {net }}$, and $T_{\|}^{\text {net}}$, respectively. The intensity of light for any arbitrary polarizer angle can be written as follows:

$$
I=T_{\|}^{\mathrm{net}} \cos ^{2}\left(\phi_{\mathrm{p}}-\phi\right)+T_{\perp}^{\mathrm{net}} \sin ^{2}\left(\phi_{\mathrm{p}}-\phi\right) .
$$

To correct the data, the measured intensity is normalized by $I$ given above for each value of the polarizer angle $\phi_{\mathrm{p}}$.

\section{Correction for rotation of polarization upon refraction}

As discussed above, when polarized light refracts from one medium to another, the transmitted intensities (or equivalently, amplitudes) differ for the components of the polarization parallel and perpendicular to the plane of incidence. Due to this difference, a small rotation of the polarization occurs upon refraction. Kamb (1958) discusses this effect at length, and concludes that this rotation should be neglected as long as the polarization suffers an identical rotation as the light is refracted upon exiting the sample. This would be true, for example, if the ice were sandwiched between two pieces of glass. In practice, however, the ice is usually cemented to a single piece of glass. Therefore, one must consider the effect of the unequal rotations of the polarization as light enters and exits the sample. Again using Fresnel's laws of refraction, it is possible to show that the rotation of the polarization is specified by the following equation (Kamb, 1958):

$$
\phi_{\mathrm{p}}^{\prime}=\phi+\tan ^{-1}\left[\tan \left(\phi_{\mathrm{p}}-\phi\right) \cos \left(\theta_{\mathrm{i}}-\theta_{\mathrm{t}}\right)\right] .
$$

$\theta_{\mathrm{i}}$ is the incident angle and $\theta_{\mathrm{t}}$ is the refracted-ray angle, both with respect to the normal to the sample. The correction required will be given by one-half the difference between the rotations for light going into and out of the sample, as follows (Kamb, 1958):

$\triangle \phi_{\mathrm{p}}($ polarization $)$

$$
\begin{aligned}
= & \frac{1}{2}\left\{\tan ^{-1}\left[\tan \left(\phi_{\mathrm{p}}^{\prime}-\phi\right) \cos \left(\theta_{1}-\theta_{2}\right) \cos \left(\theta_{2}-\theta_{3}\right)\right]\right. \\
& \left.-\tan ^{-1}\left[\tan \left(\phi_{\mathrm{p}}^{\prime}-\phi\right) \cos \left(\theta_{3}-\theta_{1}\right)\right]\right\} .
\end{aligned}
$$

$\theta_{1}, \theta_{2}$ and $\theta_{3}$ are given by the angle between the ray and the normal to the sample in each medium, as shown in Figure 9. This correction must be added to the measured polarizer extinction angle.

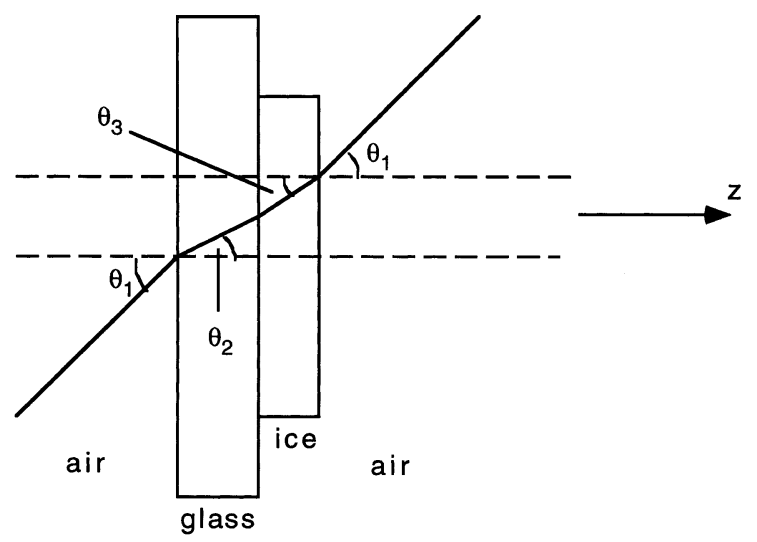

Fig. 9. Angles of refraction as light is refracted from air into glass, then glass into ice, and finally from ice back into air.

\section{Summary of corrections}

The latter three corrections (off-axis polarization, intensity variation, polarization upon rotation) are all quite small, and can be neglected if accuracy better than $1-2^{\circ}$ is not required. The first correction (tilt correction) can be very important for larger samples $(>2$ in $(5.8 \mathrm{~cm})$ diameter). For the results presented in this paper, the data were analyzed with all the corrections except for that due to intensity variations, which was difficult to apply because of the non-linearity of the CGD camera. I expect that it will be necessary to apply all four corrections to obtain high accuracy $\left(<0.5^{\circ}\right)$ for the study of large ice-core samples planned with the automated version of the apparatus. 